\title{
An Alternating Direction Method for Convex Quadratic Second-Order Cone Programming with Bounded Constraints
}

\author{
Xuewen $\mathrm{Mu}^{1}$ and Yaling Zhang ${ }^{1,2}$ \\ ${ }^{1}$ School of Mathematics and Statistics, Xidian University, Xian 710071, China \\ ${ }^{2}$ School of Computer Science, Xian Science and Technology University, Xian 710054, China \\ Correspondence should be addressed to Xuewen Mu; xdmuxuewen@hotmail.com
}

Received 20 October 2014; Revised 26 March 2015; Accepted 30 March 2015

Academic Editor: Gerhard-Wilhelm Weber

Copyright (C) $2015 \mathrm{X}$. Mu and Y. Zhang. This is an open access article distributed under the Creative Commons Attribution License, which permits unrestricted use, distribution, and reproduction in any medium, provided the original work is properly cited.

\begin{abstract}
An alternating direction method is proposed for convex quadratic second-order cone programming problems with bounded constraints. In the algorithm, the primal problem is equivalent to a separate structure convex quadratic programming over secondorder cones and a bounded set. At each iteration, we only need to compute the metric projection onto the second-order cones and the projection onto the bound set. The result of convergence is given. Numerical results demonstrate that our method is efficient for the convex quadratic second-order cone programming problems with bounded constraints.
\end{abstract}

\section{Introduction}

In this paper, we consider a convex quadratic second-order cone programming (CQSOCP) problem with bounded constraints which is defined by minimizing a convex quadratic function over the intersection of an affine set, a bounded set, and the product of second-order cones. The primal convex quadratic second-order cone programming problem is defined as

$$
\begin{array}{ll}
\min & \frac{1}{2} x^{T} Q x+c^{T} x \\
\text { s.t. } & A x=b \\
& x \in K, \quad x \in \Omega,
\end{array}
$$

where $\Omega=\{x \mid l \leq x \leq u\}$ is a bounded set, $Q$ is an $n \times n$ symmetric positive semidefinite matrix, $A \in \mathfrak{R}^{m \times n}, c \in \mathfrak{R}^{n}$, $b \in \mathfrak{R}^{m}$, and $x=\left[x_{1}, \ldots, x_{N}\right] \in \mathfrak{R}^{n_{1}} \times \cdots \times \mathfrak{R}^{n_{N}}$ is viewed as a column vector in $\Re^{n_{1}+\cdots+n_{N}}$ with $\sum_{i=1}^{N} n_{i}=n$. In addition, $K=K^{n_{1}} \times K^{n_{2}} \times \cdots \times K^{n_{N}}$ and $x_{i} \in K^{n_{i}}$, where $K^{n_{i}}$ is the $n_{i}$-dimensional second-order cone given by

$$
K^{n_{i}}=\left\{x_{i}=\left[\begin{array}{c}
x_{i_{1}} \\
x_{i_{0}}
\end{array}\right] \in \Re^{n_{i}-1} \times \Re:\left\|x_{i_{1}}\right\|_{2} \leq x_{i_{0}}\right\},
$$

where $\|\cdot\|_{2}$ is the standard Euclidean norm.
Convex quadratic second-order cone programming problem with bounded constraints is a nonlinear programming problem, which can be seen as a trust region subproblem in the trust region method for the nonlinear second-order cone programming $[1,2]$. Since $Q$ is symmetric positive semidefinite, we can compute its positive semidefinite square $\operatorname{root} Q^{1 / 2}$ by the Cholesky method. Then problem (1) can be equivalently transformed as the following mix linear and second-order cone programming (MLSOCP) [3]:

$$
\begin{aligned}
\min & t+c^{T} x \\
\text { s.t. } & A x=b \\
& \sqrt{(t-1)^{2}+2\left\|Q^{1 / 2} x\right\|_{2}^{2}} \leq t+1 \\
& l \leq x \leq u \\
& x \in K .
\end{aligned}
$$

In paper $[1,2]$, the authors use those well developed and publicly available softwares, based on interior-point methods, such as SeDuMi [4] and SDPT3 [5] to solve the equivalent MLSOCP (3).

Interior-point methods have been well developed for linear symmetric cone programming [6-8]. However, at each 
iteration these solvers require to formulate and solve a dense Schur complement matrix, which for the CQSOCP problem with bounded constraints amounts to a linear system of dimension $(m+3 n+2) \times(m+3 n+2)$. In addition, the transformed method needs to compute the square root of semidefinite matrix $Q$. When $n$ is large, because of the very large size and ill-conditioning of the linear system of equations, interior-point methods are difficult to solve the transformed MLSOCP problem efficiently [3].

The alternating direction method (ADM) has been an effective first-order approach for solving large optimization problems, such as linear programming [9], linear semidefinite programming (LSDP) $[10,11]$, nonlinear convex optimization [12], and nonsmooth $l_{1}$ minimization arising from compressive sensing $[13,14]$. A modified alternating direction method is proposed for convex quadratically constrained quadratic semidefinite programs in paper [15]. In the thesis [3], a semismooth Newton-CG augmented Lagrangian method is proposed for large scale convex quadratic symmetric cone programming. In paper [16], an alternating direction dual augmented Lagrangian method for solving linear semidefinite programming problems in standard form is presented and extended to the SDP with inequality constraints and positivity constraints.

In the paper, an alternating direction method for the CQSOCP problem with bounded constraints is proposed. Firstly, the primal problem is equivalent to a separate structure convex quadratic programming over second-order cones and a bounded set. Then the alternating direction method is proposed to solve the separate structure convex quadratic programming. In the alternating direction method, we only need to compute the metric projection onto the second-order cones and projection onto the bounded set at each iteration. We also give the convergence results and the numerical results.

\section{The Projection on the Second-Order Cone and the Bounded Set}

In this section, we will give the projection results on the second-order cones and the bounded set.

Let $x_{i}=\left[\begin{array}{l}x_{i_{1}} \\ x_{i_{0}}\end{array}\right] \in \mathfrak{R}^{n_{i}-1} \times \mathfrak{R}$ for $i=1,2, \ldots, N$; then the spectral decomposition of $x_{i}$ associate with second-order cone $K^{n_{i}}$ can be described as [17-19]

$$
x_{i}=\lambda_{1}\left(x_{i}\right) c_{1}\left(x_{i}\right)+\lambda_{2}\left(x_{i}\right) c_{2}\left(x_{i}\right), \quad i=1,2, \ldots, N
$$

where

$$
\begin{aligned}
& \lambda_{1}\left(x_{i}\right)=x_{i_{0}}-\left\|x_{i_{1}}\right\|_{2}, \\
& \lambda_{2}\left(x_{i}\right)=x_{i_{0}}+\left\|x_{i_{1}}\right\|_{2}, \\
& c_{1}\left(x_{i}\right)=\frac{1}{2}\left[\begin{array}{c}
-w \\
1
\end{array}\right], \\
& c_{2}\left(x_{i}\right)=\frac{1}{2}\left[\begin{array}{c}
w \\
1
\end{array}\right]
\end{aligned}
$$

with $w=-x_{i_{1}} /\left\|x_{i_{1}}\right\|_{2}$ if $x_{i_{1}} \neq 0$ and any vector in $\mathfrak{R}^{n_{i}-1}$ satisfying $\|w\|_{2}=1$ if $x_{i_{1}}=0$.

Next we introduce the projection lemma over the secondorder cone [17-19].

Lemma 1 (see [17-19]). For any $x_{i}=\left[\begin{array}{l}x_{i_{1}} \\ x_{i_{0}}\end{array}\right] \in \mathfrak{R}^{n_{i}-1} \times \mathfrak{R}$, let $P_{K^{n_{i}}}\left(x_{i}\right)$ be the projection of $x_{i}$ onto the second-order cone $K^{n_{i}}$; then we have

$$
\begin{array}{r}
P_{K^{n_{i}}}\left(x_{i}\right)=\left(\lambda_{1}\left(x_{i}\right)\right)_{+} c_{1}\left(x_{i}\right)+\left(\lambda_{2}\left(x_{i}\right)\right)_{+} c_{2}\left(x_{i}\right), \\
i=1,2, \ldots, N,
\end{array}
$$

where $s_{+}:=\max (0, s)$ for $s \in \mathfrak{R}$.

Let $x=\left[x_{1}, \ldots, x_{N}\right] \in \Re^{n_{1}} \times \cdots \times \Re^{n_{N}}$; then the projection $P_{K}(x)$ of $x$ over the cone $K$ is described as

$$
\begin{aligned}
P_{K}(x) & =\left[P_{K^{n_{1}}}\left(x_{1}\right), \ldots, P_{K^{n_{N}}}\left(x_{N}\right)\right] \\
& \in \mathfrak{R}^{n_{1}} \times \cdots \times \mathfrak{R}^{n_{N}} .
\end{aligned}
$$

Let $y \in \mathfrak{R}^{n}$; then the projection on the bounded set $\Omega$ is easy to carry out, namely, through an element by element method:

$$
P_{\Omega}(y)=\max \{l, \min \{y, u\}\}
$$

\section{An Alternating Direction Method for CQSOCP Problems with Bounded Constraints}

In this section, we give an alternating direction method for convex quadratic second-order cone programming problems with bounded constraints.

Firstly, we give an equivalent separate structure convex quadratic programming over second-order cone and bounded set as follows:

$$
\begin{array}{ll}
\min & \frac{1}{2} x^{T} Q x+c^{T} y \\
\text { s.t. } & A x=b, x \in K \\
& x=y, \quad y \in \Omega .
\end{array}
$$

The Lagrangian function for the separate structure convex quadratic programming problem is written as

$$
\begin{aligned}
\max _{\lambda, \mu} \min _{x \in K, y \in \Omega} L(x, y, \lambda, \mu) \\
=\frac{1}{2} x^{T} Q x+c^{T} y-\lambda^{T}(A x-b)-\mu^{T}(x-y),
\end{aligned}
$$

where $\lambda \in \mathfrak{R}^{m}, \mu \in R^{n}$.

Under mild constraint qualifications (e.g., Slater condition), strong duality holds for problem (9), and hence, $x^{*}$ is an optimal solution of (9) if and only if there exists 
$\left(x^{*}, y^{*}, \lambda^{*}, \mu^{*}\right) \in K \times \Omega \times \mathfrak{R}^{m} \times \mathfrak{R}^{n}$ satisfying the following KKT system in variational inequality form:

$$
\begin{aligned}
\left\langle x-x^{*}, Q x^{*}-A^{T} \lambda^{*}-\mu^{*}\right\rangle & \geq 0, \quad \forall x \in K, \\
\left\langle y-y^{*}, c+\mu^{*}\right\rangle & \geq 0, \quad \forall y \in \Omega, \\
A x^{*} & =b, \\
x^{*} & =y^{*} .
\end{aligned}
$$

The augmented Lagrangian function for the the separate structure convex quadratic programming problem is defined as

$$
\begin{aligned}
L(x, y, \lambda, \mu)= & \frac{1}{2} x^{T} Q x+c^{T} y-\lambda^{T}(A x-b) \\
& -\mu^{T}(x-y)+\frac{1}{2 \beta_{1}}\|A x-b\|_{2}^{2} \\
& +\frac{1}{2 \beta_{2}}\|x-y\|_{2}^{2},
\end{aligned}
$$

where $\beta_{1}, \beta_{2}>0$.

The variational inequality form of alternating direction method for (12) is as follows.

3.1. The Original Alternating Direction Method. Given $x^{0} \in$ $K, y^{0} \in \Omega, \lambda^{0} \in \mathfrak{R}^{m}, \mu^{0} \in \mathfrak{R}^{n}$, and $\beta_{1}, \beta_{2}>0$. For $k=$ $0,1,2, \ldots$, then consider the following.

Step 1. Consider $\left(x^{k}, y^{k}, \lambda^{k}, \mu^{k}\right) \rightarrow\left(x^{k+1}, y^{k}, \lambda^{k}, \mu^{k}\right)$; we compute $x^{k+1}$, which satisfies

$$
\begin{aligned}
& \left\langle x-x^{k+1}, Q x^{k+1}-A^{T} \lambda^{k}-\mu^{k}+\frac{1}{\beta_{1}} A^{T}\left(A x^{k+1}-b\right)\right. \\
& \left.+\frac{1}{\beta_{2}}\left(x^{k+1}-y^{k}\right)\right\rangle \geq 0, \quad \forall x \in K .
\end{aligned}
$$

Step 2. Consider $\left(x^{k+1}, y^{k}, \lambda^{k}, \mu^{k}\right) \rightarrow\left(x^{k+1}, y^{k+1}, \lambda^{k}, \mu^{k}\right)$; we compute $y^{k+1}$, which satisfies

$$
\left\langle y-y^{k+1}, c+\mu^{k}-\frac{1}{\beta_{2}}\left(x^{k+1}-y^{k+1}\right)\right\rangle \geq 0,
$$

$\forall y \in \Omega$.

Step 3. Consider $\left(x^{k+1}, y^{k+1}, \lambda^{k}, \mu^{k}\right) \rightarrow\left(x^{k+1}, y^{k+1}, \lambda^{k+1}\right.$, $\left.\mu^{k}\right)$; update the Lagrange multiplier by

$$
\lambda^{k+1}=\lambda^{k}-\frac{1}{\beta_{1}}\left(A x^{k+1}-b\right) \text {. }
$$

Step 4. Consider $\left(x^{k+1}, y^{k+1}, \lambda^{k+1}, \mu^{k}\right) \rightarrow\left(x^{k+1}, y^{k+1}, \lambda^{k+1}\right.$, $\left.\mu^{k+1}\right)$; update the Lagrange multiplier by

$$
\mu^{k+1}=\mu^{k}-\frac{1}{\beta_{2}}\left(x^{k+1}-y^{k+1}\right) .
$$

In Steps 1 and 2, we should solve variational inequalities. In the following analysis, we will convert them to simple projection operations.

Lemma 2 (see [20]). Let $\Theta$ be a closed convex set in a Hilbert space and let $P_{\Theta}(x)$ be the projection of $x$ onto $\Theta$. Then

$$
\langle z-y, y-x\rangle \geq 0, \quad \forall z \in \Theta \Longleftrightarrow y=P_{\Theta}(x) .
$$

Taking $x=x^{k+1}-\alpha_{1}\left(Q x^{k+1}-A^{T} \lambda^{k}-\mu^{k}+\right.$ $\left.\left(1 / \beta_{1}\right) A^{T}\left(A x^{k+1}-b\right)+\left(1 / \beta_{2}\right)\left(x^{k+1}-y^{k}\right)\right)$ and $y=x^{k+1}$ in (17), we see that (13) is equivalent to the following nonlinear equation:

$$
\begin{aligned}
x^{k+1} & =P_{K}\left[x^{k+1}-\alpha_{1}\left(Q x^{k+1}-A^{T} \lambda^{k}-\mu^{k}\right.\right. \\
+ & \left.\left.\frac{1}{\beta_{1}} A^{T}\left(A x^{k+1}-b\right)+\frac{1}{\beta_{2}}\left(x^{k+1}-y^{k}\right)\right)\right],
\end{aligned}
$$

where $\alpha_{1}$ can be any positive number.

Taking $x=y^{k+1}-\alpha_{2}\left(c+\mu^{k}-\left(1 / \beta_{2}\right)\left(x^{k+1}-y^{k+1}\right)\right)$ and $y=y^{k+1}$ in (17), we see that (14) is equivalent to the following nonlinear equation:

$$
y^{k+1}=P_{\Omega}\left[y^{k+1}-\alpha_{2}\left(c+\mu^{k}-\frac{1}{\beta_{2}}\left(x^{k+1}-y^{k+1}\right)\right)\right],
$$

where $\alpha_{2}$ can be any positive number.

Due to the existence of the terms $Q x^{k+1}$ and $A^{T} A x^{k+1}$ in (18), we can not compute $x^{k+1}$ directly. We therefore use the following approximate approach which is similar to the one in paper [15]. For certain constants $\gamma_{1}$ and $\gamma_{2}$, let

$$
\begin{aligned}
& R_{1}\left(x^{k}, x^{k+1}\right)=Q x^{k+1}-Q x^{k}-\gamma_{1}\left(x^{k+1}-x^{k}\right), \\
& R_{2}\left(x^{k}, x^{k+1}\right)=A^{T} A x^{k+1}-A^{T} A x^{k}-\gamma_{2}\left(x^{k+1}-x^{k}\right)
\end{aligned}
$$

be the residual between $Q x^{k+1}, A^{T} A x^{k+1}$ and their linearization at $x^{k}$, respectively.

Instead of computing (18), we compute

$$
\begin{aligned}
& x^{k+1}=P_{K}\left[x^{k+1}-\alpha_{1}\left(Q x^{k+1}-A^{T} \lambda^{k}-\mu^{k}\right.\right. \\
& +\frac{1}{\beta_{1}} A^{T}\left(A x^{k+1}-b\right)+\frac{1}{\beta_{2}}\left(x^{k+1}-y^{k}\right) \\
& \left.\left.\quad-R_{1}\left(x^{k}, x^{k+1}\right)-\frac{1}{\beta_{1}} R_{2}\left(x^{k}, x^{k+1}\right)\right)\right]=P_{K}\left[x^{k+1}\right. \\
& -\alpha_{1}\left(\left(\frac{1}{\beta_{2}}+\gamma_{1}+\frac{\gamma_{2}}{\beta_{1}}\right) x^{k+1}-A^{T} \lambda^{k}-\mu^{k}-\frac{1}{\beta_{1}} A^{T} b\right. \\
& \left.\left.-\frac{1}{\beta_{2}} y^{k}+Q x^{k}-\gamma_{1} x^{k}+\frac{1}{\beta_{1}} A^{T} A x^{k}-\frac{\gamma_{2}}{\beta_{1}} x^{k}\right)\right] .
\end{aligned}
$$

We choose $\gamma_{1}, \gamma_{2}$ so that $\gamma_{1}>\lambda_{\max }(Q), \gamma_{2}>\lambda_{\max }\left(A^{T} A\right)$, where $\lambda_{\max }(Q)$ and $\lambda_{\text {max }}\left(A^{T} A\right)$ are the largest eigenvalues of $Q$ and $A^{T} A$, respectively. 
Setting

$$
\alpha_{1}=\left(\frac{1}{\beta_{2}}+\gamma_{1}+\frac{\gamma_{2}}{\beta_{1}}\right)^{-1}
$$

in (21), we have

$$
\begin{aligned}
& x^{k+1}=P_{K}\left[\alpha _ { 1 } \left(A^{T} \lambda^{k}+\mu^{k}+\frac{1}{\beta_{1}} A^{T} b+\frac{1}{\beta_{2}} y^{k}-Q x^{k}\right.\right. \\
& \left.\left.+\gamma_{1} x^{k}-\frac{1}{\beta_{1}} A^{T} A x^{k}+\frac{\gamma_{2}}{\beta_{1}} x^{k}\right)\right],
\end{aligned}
$$

which will be used as an approximation to the solution of variational inequality (13).

Let $\alpha_{2}=\beta_{2}$ in (19); we have

$$
y^{k+1}=P_{\Omega}\left[-\alpha_{2}\left(c+\mu^{k}-\frac{1}{\beta_{2}} x^{k+1}\right)\right] .
$$

In summary, the modified alternating direction method is given as follows.

3.2. The Modified Alternating Direction Method. Given $x^{0} \in$ $K, y^{0} \in \Omega, \lambda^{0} \in \mathfrak{R}^{m}, \mu^{0} \in \mathfrak{R}^{n}$, and $\beta_{1}, \beta_{2}>0$. For $k=$ $0,1,2, \ldots$, then consider the following.

Step 1. Consider $\left(x^{k}, y^{k}, \lambda^{k}, \mu^{k}\right) \rightarrow\left(x^{k+1}, y^{k}, \lambda^{k}, \mu^{k}\right)$; we compute $x^{k+1}$, which satisfies

$$
\begin{gathered}
x^{k+1}=P_{K}\left[( \frac { 1 } { \beta _ { 2 } } + \gamma _ { 1 } + \frac { \gamma _ { 2 } } { \beta _ { 1 } } ) ^ { - 1 } \left(A^{T} \lambda^{k}+\mu^{k}+\frac{1}{\beta_{1}} A^{T} b\right.\right. \\
\left.\left.+\frac{1}{\beta_{2}} y^{k}-Q x^{k}+\gamma_{1} x^{k}-\frac{1}{\beta_{1}} A^{T} A x^{k}+\frac{\gamma_{2}}{\beta_{1}} x^{k}\right)\right] .
\end{gathered}
$$

Step 2. Consider $\left(x^{k+1}, y^{k}, \lambda^{k}, \mu^{k}\right) \rightarrow\left(x^{k+1}, y^{k+1}, \lambda^{k}, \mu^{k}\right)$; we compute $y^{k+1}$, which satisfies

$$
y^{k+1}=P_{\Omega}\left[x^{k+1}-\beta_{2}\left(c+\mu^{k}\right)\right] .
$$

Step 3. Consider $\left(x^{k+1}, y^{k+1}, \lambda^{k}, \mu^{k}\right) \rightarrow\left(x^{k+1}, y^{k+1}, \lambda^{k+1}\right.$, $\left.\mu^{k}\right)$; update the Lagrange multiplier by

$$
\lambda^{k+1}=\lambda^{k}-\frac{1}{\beta_{1}}\left(A x^{k+1}-b\right) \text {. }
$$

Step 4. Consider $\left(x^{k+1}, y^{k+1}, \lambda^{k+1}, \mu^{k}\right) \rightarrow\left(x^{k+1}, y^{k+1}, \lambda^{k+1}\right.$, $\left.\mu^{k+1}\right)$; update the Lagrange multiplier by

$$
\mu^{k+1}=\mu^{k}-\frac{1}{\beta_{2}}\left(x^{k+1}-y^{k+1}\right) .
$$

From Steps 1 and 2, the modified alternating direction method only needs to compute the metric projection of vectors onto $K$ and $\Omega$. From Steps 3 and 4, we could interpret $1 / \beta_{1}$ and $1 / \beta_{2}$ as the dual stepsizes. Therefore, the iteration of our method is simple and fast.

\section{The Convergence Result}

In this section, we extended and modified the convergence results of the alternating direction methods for convex quadratically constrained quadratic semidefinite programs in paper [15] and then give the convergence analysis of the alternating direction method for CQSOCP problems with bounded constraints.

Lemma 3. The sequence $\left\{x^{k}, y^{k}, \lambda^{k}, \mu^{k}\right\}$ generated by the modified alternating direction method satisfies

$$
\begin{aligned}
& \left\langle x^{k+1}-x^{*}, R_{1}\left(x^{k}, x^{k+1}\right)+\frac{1}{\beta_{1}} R_{2}\left(x^{k}, x^{k+1}\right)\right\rangle \\
& +\frac{1}{\beta_{2}}\left\langle y^{k+1}-y^{*}, y^{k}-y^{k+1}\right\rangle \\
& +\beta_{1}\left\langle\lambda^{k+1}-\lambda^{*}, \lambda^{k}-\lambda^{k+1}\right\rangle \\
& +\beta_{2}\left\langle\mu^{k+1}-\mu^{*}, \mu^{k}-\mu^{k+1}\right\rangle \geq 0,
\end{aligned}
$$

where $\left\{x^{*}, y^{*}, \lambda^{*}, \mu^{*}\right\}$ is a KKT point of system (11).

Proof. Let $y=y^{k+1}$ in the second inequality in system (11); we have

$$
\left\langle y^{k+1}-y^{*}, c+\mu^{*}\right\rangle \geq 0 .
$$

Let $y=y^{*}$ in (14), and coupled with (16), we have

$$
\left\langle y^{*}-y^{k+1}, c+\mu^{k+1}\right\rangle \geq 0 .
$$

Adding (30) and (31) together, we have

$$
\left\langle y^{k+1}-y^{*}, \mu^{*}-\mu^{k+1}\right\rangle \geq 0 .
$$

In addition, from (14) and (16), we have

$$
\begin{aligned}
\left\langle y^{k}-y^{k+1}, c+\mu^{k+1}\right\rangle & \geq 0, \\
\left\langle y^{k+1}-y^{k}, c+\mu^{k}\right\rangle & \geq 0 .
\end{aligned}
$$

Adding the two inequality above, we have

$$
\left\langle y^{k+1}-y^{k}, \mu^{k}-\mu^{k+1}\right\rangle \geq 0 .
$$

Note that (21) can be written equivalently as

$$
\begin{aligned}
& \left\langle x-x^{k+1}, Q x^{k+1}-A^{T} \lambda^{k+1}-\mu^{k+1}+\frac{1}{\beta_{2}}\left(y^{k+1}-y^{k}\right)\right. \\
& \left.-R_{1}\left(x^{k}, x^{k+1}\right)-\frac{1}{\beta_{1}} R_{2}\left(x^{k}, x^{k+1}\right)\right\rangle \geq 0,
\end{aligned}
$$

$\forall x \in K$

Setting $x=x^{*}$, we have

$$
\begin{aligned}
& \left\langle x^{*}-x^{k+1}, Q x^{k+1}-A^{T} \lambda^{k+1}-\mu^{k+1}+\frac{1}{\beta_{2}}\left(y^{k+1}-y^{k}\right)\right. \\
& \left.-R_{1}\left(x^{k}, x^{k+1}\right)-\frac{1}{\beta_{1}} R_{2}\left(x^{k}, x^{k+1}\right)\right\rangle \geq 0 .
\end{aligned}
$$


Let $x=x^{k+1}$ in the first inequality in system (11); we have

$$
\left\langle x^{k+1}-x^{*}, Q x^{*}-A^{T} \lambda^{*}-\mu^{*}\right\rangle \geq 0 .
$$

Adding (36) and (37) together, we have

$$
\begin{aligned}
& \left\langle x^{k+1}-x^{*}, A^{T}\left(\lambda^{k+1}-\lambda^{*}\right)\right\rangle \\
& +\left\langle x^{k+1}-x^{*}, \mu^{k+1}-\mu^{*}\right\rangle \\
& +\left\langle x^{k+1}-x^{*}, \frac{1}{\beta_{2}}\left(y^{k}-y^{k+1}\right)\right\rangle \\
& +\left\langle x^{k+1}-x^{*}, R_{1}\left(x^{k}, x^{k+1}\right)+\frac{1}{\beta_{1}} R_{2}\left(x^{k}, x^{k+1}\right)\right\rangle \\
& \geq\left\langle x^{k+1}-x^{*}, Q\left(x^{k+1}-x^{*}\right)\right\rangle \geq 0 .
\end{aligned}
$$

From first part at the left side of (38) and the third equation in system (11), we have

$$
\begin{aligned}
& \left\langle x^{k+1}-x^{*}, A^{T}\left(\lambda^{k+1}-\lambda^{*}\right)\right\rangle \\
& =\left\langle A x^{k+1}-A x^{*}, \lambda^{k+1}-\lambda^{*}\right\rangle \\
& =\left\langle A x^{k+1}-b, \lambda^{k+1}-\lambda^{*}\right\rangle \\
& =\beta_{1}\left\langle\lambda^{k+1}-\lambda^{*}, \lambda^{k}-\lambda^{k+1}\right\rangle .
\end{aligned}
$$

From (16), (36), the last equation in system (11), and the second part at the left side of (38), we have

$$
\begin{aligned}
& \left\langle x^{k+1}-x^{*}, \mu^{k+1}-\mu^{*}\right\rangle+\left\langle y^{k+1}-y^{*}, \mu^{*}-\mu^{k+1}\right\rangle \\
& =\left\langle\mu^{k+1}-\mu^{*}, x^{k+1}-x^{*}-y^{k+1}+y^{*}\right\rangle \\
& =\left\langle\mu^{k+1}-\mu^{*}, x^{k+1}-y^{k+1}\right\rangle \\
& =\beta_{2}\left\langle\mu^{k+1}-\mu^{*}, \mu^{k}-\mu^{k+1}\right\rangle .
\end{aligned}
$$

In addition, from the third part at the left side of (38), we have

$$
\begin{aligned}
\frac{1}{\beta_{2}}\left\langle x^{k+1}-x^{*}, y^{k}-y^{k+1}\right\rangle \\
=\frac{1}{\beta_{2}}\left\langle y^{k+1}-y^{*}, y^{k}-y^{k+1}\right\rangle \\
\quad+\frac{1}{\beta_{2}}\left\langle x^{k+1}-y^{k+1}, y^{k}-y^{k+1}\right\rangle \\
=\frac{1}{\beta_{2}}\left\langle y^{k+1}-y^{*}, y^{k}-y^{k+1}\right\rangle \\
\quad-\left\langle y^{k+1}-y^{k}, \mu^{k}-\mu^{k+1}\right\rangle .
\end{aligned}
$$

It follows from (32)-(34) and (38)-(41) that

$$
\begin{aligned}
& \left\langle x^{k+1}-x^{*}, R_{1}\left(x^{k}, x^{k+1}\right)+\frac{1}{\beta_{1}} R_{2}\left(x^{k}, x^{k+1}\right)\right\rangle \\
& +\frac{1}{\beta_{2}}\left\langle y^{k+1}-y^{*}, y^{k}-y^{k+1}\right\rangle \\
& +\beta_{1}\left\langle\lambda^{k+1}-\lambda^{*}, \lambda^{k}-\lambda^{k+1}\right\rangle \\
& +\beta_{2}\left\langle\mu^{k+1}-\mu^{*}, \mu^{k}-\mu^{k+1}\right\rangle \geq 0 .
\end{aligned}
$$

Now, we give the convergent conclusion.

Theorem 4. The sequence $\left\{x^{k}\right\}$ generated by the modified alternating direction method converges to a solution point $x^{*}$ of problem (9).

Proof. We denote

$$
w=\left[\begin{array}{l}
x \\
y \\
\lambda \\
\mu
\end{array}\right],
$$

$$
=\left[\begin{array}{cccc}
\left(\gamma_{1} I_{n}-Q\right)+\frac{1}{\beta_{1}}\left(\gamma_{2} I_{n}-A^{T} A\right) & 0 & 0 & 0 \\
0 & \frac{1}{\beta_{2}} I_{n} & 0 & 0 \\
0 & 0 & \beta_{1} I_{m} & 0 \\
0 & 0 & 0 & \beta_{2} I_{n}
\end{array}\right],
$$

where $I_{n}$ denotes the $n$-dimensional unit matrix and $G$ is positive definite. Here, we define the $G$-inner product of $w$ and $\bar{w}$ as

$$
\begin{aligned}
\langle w, \bar{w}\rangle_{G}= & \left\langle x,\left(\gamma_{1} I_{n}-Q\right) \bar{x}+\frac{1}{\beta_{1}}\left(\gamma_{2} I_{n}-A^{T} A\right) \bar{x}\right\rangle \\
& +\frac{1}{\beta_{2}}\langle y, \bar{y}\rangle+\beta_{1}\langle\lambda, \bar{\lambda}\rangle+\beta_{2}\langle\mu, \bar{\mu}\rangle
\end{aligned}
$$

and the associated $G$-norm as

$$
\begin{aligned}
& \|w\|_{G}=\left\{\|x\|_{\left(\gamma_{1} I_{n}-Q\right)+\left(1 / \beta_{1}\right)\left(\gamma_{2} I_{n}-A^{T} A\right)}^{2}+\frac{1}{\beta_{2}}\|y\|_{2}^{2}\right. \\
& \left.+\beta_{1}\|\lambda\|_{2}^{2}+\beta_{2}\|\mu\|_{2}^{2}\right\}^{0.5},
\end{aligned}
$$

where $\|x\|_{\left(\gamma_{1} I_{n}-Q\right)+\left(1 / \beta_{1}\right)\left(\gamma_{2} I_{n}-A^{T} A\right)}^{2}=x^{T}\left\{\left(\gamma_{1} I_{n}-Q\right)+\left(1 / \beta_{1}\right)\right.$ $\left.\left(\gamma_{2} I_{n}-A^{T} A\right)\right\} x$. 
Observe that, by Lemma 2, solving the optimal condition (11) for problem (9) is equivalent to finding a zero point of the residual function:

$$
e(w)=\left\|\begin{array}{c}
x-P_{K}\left(x-\alpha_{1}\left(Q x-A^{T} \lambda-\mu\right)\right) \\
y-P_{\Omega}\left(y-\alpha_{2}(c+\mu)\right) \\
A x-b \\
x-y
\end{array}\right\|_{2}
$$

From (15), (16), and the first equation in (21), we have that

$$
\begin{aligned}
x^{k+1} & =P_{K}\left[x^{k+1}-\alpha_{1}\left(Q x^{k+1}-A^{T} \lambda^{k+1}-\mu^{k+1}\right.\right. \\
& +\frac{1}{\beta_{2}}\left(y^{k+1}-y^{k}\right)-R_{1}\left(x^{k}, x^{k+1}\right) \\
& \left.\left.-\frac{1}{\beta_{1}} R_{2}\left(x^{k}, x^{k+1}\right)\right)\right] .
\end{aligned}
$$

From (19) and (16), we have

$$
\begin{aligned}
y^{k+1} & =P_{\Omega}\left[y^{k+1}-\alpha_{2}\left(c+\mu^{k}-\frac{1}{\beta_{2}}\left(x^{k+1}-y^{k+1}\right)\right)\right] \\
& =P_{\Omega}\left[y^{k+1}-\alpha_{2}\left(c+\mu^{k+1}\right)\right] .
\end{aligned}
$$

Based on (47)-(48), (15)-(16), and the nonexpansion property of the projection operator, we have

$$
\begin{aligned}
& \left\|e\left(w^{k+1}\right)\right\|_{2} \\
& \leq\left\|\begin{array}{c}
\frac{\alpha_{1}}{\beta_{2}}\left(y^{k}-y^{k+1}\right)+ \\
\alpha_{1} R_{1}\left(x^{k}, x^{k+1}\right)+\frac{\alpha_{1}}{\beta_{1}} R_{2}\left(x^{k}, x^{k+1}\right) \\
0 \\
\beta_{1}\left(\lambda^{k}-\lambda^{k+1}\right) \\
\beta_{2}\left(\mu^{k}-\mu^{k+1}\right)
\end{array}\right\|_{2} \\
& \leq\left\|\begin{array}{c}
\alpha_{1} R_{1}\left(x^{k}, x^{k+1}\right)+\frac{\alpha_{1}}{\beta_{1}} R_{2}\left(x^{k}, x^{k+1}\right) \\
0 \\
\beta_{1}\left(\lambda^{k}-\lambda^{k+1}\right) \\
\beta_{2}\left(\mu^{k}-\mu^{k+1}\right)
\end{array}\right\|_{2} \\
& +\left\|\begin{array}{c}
\frac{\alpha_{1}}{\beta_{2}}\left(y^{k}-y^{k+1}\right) \\
0 \\
0 \\
0
\end{array}\right\|_{2} \leq \delta\left\|w^{k}-w^{k+1}\right\|_{G},
\end{aligned}
$$

where $\delta$ is a positive constant depending on parameters $\alpha_{1}, \beta_{1}, \beta_{2}, \gamma_{1}, \gamma_{2}$, and the largest eigenvalue of $Q$ and $A^{T} A$, for example, setting

$\delta$

$$
=\sqrt{\max \left\{\beta_{1}, \beta_{2}, \frac{\alpha_{1}^{2}}{\beta_{2}}, \alpha_{1}^{2} \lambda_{\max }\left(\gamma_{1} I_{n}-Q+\frac{1}{\beta_{1}}\left(\gamma_{2} I_{n}-A^{T} A\right)\right)\right\}} .
$$

TABLE 1: The test problems with small scale.

\begin{tabular}{lcccc}
\hline Problems & $m$ & $n$ & SOC & Ratio \\
\hline P01 & 40 & 100 & $1 \times 100$ & $1.00 \%$ \\
P02 & 40 & 100 & $1 \times 40 ; 20 \times 3$ & $21.00 \%$ \\
P03 & 40 & 100 & $20 \times 5$ & $20.00 \%$ \\
P04 & 40 & 100 & $1 \times 4 ; 32 \times 3$ & $33.00 \%$ \\
\hline P05 & 120 & 200 & $1 \times 200$ & $0.50 \%$ \\
P06 & 120 & 200 & $1 \times 100 ; 1 \times 4 ; 32 \times 3$ & $17.00 \%$ \\
P07 & 120 & 200 & $40 \times 5$ & $20.00 \%$ \\
P08 & 120 & 200 & $1 \times 5 ; 65 \times 3$ & $33.00 \%$ \\
\hline P09 & 200 & 400 & $1 \times 400$ & $0.25 \%$ \\
P10 & 200 & 400 & $1 \times 200 ; 1 \times 5 ; 65 \times 3$ & $16.75 \%$ \\
P11 & 200 & 400 & $80 \times 5$ & $20.00 \%$ \\
P12 & 200 & 400 & $1 \times 4 ; 132 \times 3$ & $33.25 \%$ \\
\hline P13 & 300 & 600 & $1 \times 600$ & $0.16 \%$ \\
P14 & 300 & 600 & $1 \times 400 ; 1 \times 5 ; 65 \times 3$ & $11.16 \%$ \\
P15 & 300 & 600 & $120 \times 5$ & $20.00 \%$ \\
P16 & 300 & 600 & $200 \times 3$ & $33.33 \%$ \\
\hline
\end{tabular}

From Lemma 3, we can write (29) as

$$
\left\langle w^{k+1}-w^{*}, w^{k}-w^{k+1}\right\rangle_{G} \geq 0,
$$

which implies that

$$
\left\langle w^{k}-w^{*}, w^{k}-w^{k+1}\right\rangle_{G} \geq\left\|w^{k}-w^{k+1}\right\|_{G} .
$$

Thus

$$
\begin{aligned}
\left\|w^{k+1}-w^{*}\right\|_{G}^{2}= & \left\|\left(w^{k}-w^{*}\right)-\left(w^{k}-w^{k+1}\right)\right\|_{G}^{2} \\
= & \left\|w^{k}-w^{*}\right\|_{G}^{2} \\
& -2\left\langle w^{k}-w^{*}, w^{k}-w^{k+1}\right\rangle_{G} \\
& +\left\|w^{k}-w^{k+1}\right\|_{G}^{2} \\
\leq & \left\|w^{k}-w^{*}\right\|_{G}^{2}-\left\|w^{k}-w^{k+1}\right\|_{G}^{2} \\
\leq & \left\|w^{k}-w^{*}\right\|_{G}^{2}-\frac{1}{\delta^{2}}\left\|e\left(w^{k+1}\right)\right\|_{2}^{2} .
\end{aligned}
$$

From the above inequality, we have

$$
\left\|w^{k+1}-w^{*}\right\|_{G}^{2} \leq\left\|w^{k}-w^{*}\right\|_{G}^{2}, \quad k=1,2, \ldots .
$$

That is, the sequence $\left\{w^{k}\right\}$ is bounded. Thus there exists at least one cluster point of $\left\{w_{k}\right\}$.

It also follows from (53) that

$$
\sum_{k=0}^{\infty} \frac{1}{\delta^{2}}\left\|e\left(w^{k+1}\right)\right\|_{2}^{2}<+\infty
$$

and thus

$$
\lim _{k \rightarrow \infty}\left\|e\left(w^{k+1}\right)\right\|_{2}=0
$$


TABLE 2: The results for the test problems with small scale.

\begin{tabular}{|c|c|c|c|c|c|c|}
\hline \multirow{2}{*}{ Problems } & \multicolumn{3}{|c|}{ MADM } & \multicolumn{3}{|c|}{ SeDuMi } \\
\hline & Iter. & Time & Value & Iter. & Time & Value \\
\hline P01 & 216 & 0.14 & 6.8296723 & 9 & 0.34 & 6.8296742 \\
\hline P02 & 245 & 0.25 & 71.9788074 & 9 & 0.31 & 71.9788099 \\
\hline P03 & 264 & 0.30 & 76.2533942 & 11 & 0.39 & 76.2533927 \\
\hline P04 & 261 & 0.34 & 143.5300081 & 11 & 0.44 & 143.5300023 \\
\hline P05 & 269 & 0.33 & 47.1664568 & 10 & 0.81 & 47.1664516 \\
\hline P06 & 271 & 0.56 & 164.0914031 & 12 & 1.16 & 164.0914052 \\
\hline P07 & 293 & 0.66 & 467.8962158 & 13 & 1.28 & 467.8962123 \\
\hline P08 & 320 & 0.89 & 671.0620751 & 13 & 1.34 & 671.0620812 \\
\hline P09 & 322 & 1.53 & 71.2857981 & 12 & 5.19 & 71.2857926 \\
\hline $\mathrm{P} 10$ & 337 & 2.28 & 85.4731057 & 13 & 7.41 & 85.4731061 \\
\hline P11 & 304 & 2.14 & 1085.3976583 & 15 & 7.94 & 1085.3976475 \\
\hline P12 & 316 & 2.62 & 2256.4765633 & 16 & 10.11 & 2256.4765646 \\
\hline $\mathrm{P} 13$ & 351 & 4.17 & 89.3228874 & 11 & 15.03 & 89.3228832 \\
\hline P14 & 377 & 5.14 & 113.5586948 & 12 & 16.16 & 113.5586923 \\
\hline P15 & 327 & 5.13 & 2198.2136742 & 16 & 27.53 & 2198.2136739 \\
\hline P16 & 326 & 5.81 & 2727.7797204 & 16 & 32.67 & 2727.7797233 \\
\hline
\end{tabular}

Let $\bar{w}$ be a cluster point of $\left\{w^{k}\right\}$ and the subsequence $\left\{w^{k_{j}}\right\}$ converges to $\bar{w}$. We have

$$
\|e(\bar{w})\|_{2}=\lim _{j \rightarrow \infty}\left\|e\left(w^{k_{j}}\right)\right\|_{2}=0,
$$

so $\bar{w}$ satisfies system (11). Setting $w^{*}=\bar{w}$, we have

$$
\left\|w^{k+1}-\bar{w}\right\|_{G} \leq\left\|w^{k}-\bar{w}\right\|_{G} .
$$

The sequence $\left\{w^{k}\right\}$ satisfies

$$
\lim _{k \rightarrow \infty} w^{k}=\bar{w}
$$

\section{Simulation Experiments}

In this section we present computational results by comparing the modified alternating direction method with the interior-point method. The interior-point method is used to solve the transformed mix linear and second-order cone programming problems (3). All the algorithms are run in the MATLAB 7.0 environment on an Inter Core processor $1.80 \mathrm{GHz}$ personal computer with $2.00 \mathrm{~GB}$ of Ram.

The test problems are formulated by random method as follows:

(1) Given the values of $n, m, N, n_{i}, i=1,2, \ldots, N$ with $\sum_{i=0}^{N} n_{i}=n$.

(2) Generate a random matrix $\widetilde{Q} \in R^{n \times n}$, and set $Q=$ $\widetilde{Q}^{T} \widetilde{Q}$. At the same time, generate a random matrix $A \in$ $R^{m \times n}$ with full row rank.

(3) Set $l=-e, u=e$, where $e$ is a vector whose components are all ones.
TABLE 3: The test problems with medium scale.

\begin{tabular}{lcccc}
\hline Problems & $m$ & $n$ & SOC & Ratio \\
\hline P21 & 400 & 1000 & $100 \times 10$ & $10.00 \%$ \\
P22 & 400 & 1000 & $1 \times 200 ; 160 \times 5$ & $16.10 \%$ \\
P23 & 400 & 1000 & $1 \times 4 ; 332 \times 3$ & $33.30 \%$ \\
\hline P24 & 600 & 2000 & $50 \times 40$ & $2.5 \%$ \\
P25 & 600 & 2000 & $1 \times 400 ; 1 \times 4 ; 532 \times 3$ & $26.70 \%$ \\
P26 & 600 & 2000 & $1 \times 5 ; 665 \times 3$ & $33.33 \%$ \\
\hline P27 & 800 & 3000 & $100 \times 30$ & $3.33 \%$ \\
P28 & 800 & 3000 & $1 \times 600 ; 800 \times 3$ & $26.70 \%$ \\
P29 & 800 & 3000 & $1000 \times 3$ & $33.33 \%$ \\
\hline P30 & 1000 & 4000 & $100 \times 40$ & $2.50 \%$ \\
P31 & 1000 & 4000 & $1 \times 200 ; 760 \times 5$ & $19.02 \%$ \\
P32 & 1000 & 4000 & $1 \times 4 ; 1332 \times 3$ & $33.32 \%$ \\
\hline P33 & 2000 & 5000 & $100 \times 50$ & $2.00 \%$ \\
P34 & 2000 & 5000 & $1 \times 400 ; 920 \times 5$ & $18.42 \%$ \\
P45 & 2000 & 5000 & $1 \times 5 ; 1665 \times 3$ & $33.32 \%$ \\
\hline
\end{tabular}

(4) Given $x=\left[x_{1}, x_{2}, \ldots, x_{N}\right] \in \mathfrak{R}^{n_{1}} \times \cdots \times \mathfrak{R}^{n_{N}}$, then generate the random vector $x_{i} \in\left\{l_{i}, u_{i}\right\}$ and make it an interior point of second-order cone $K^{n_{i}}$ for $i=$ $1,2, \ldots, N$.

(5) We obtain $b$ by computing $b=A x$.

The first set of test problems includes 16 small scale CQSOCP problems with bounded constraints, which is shown in Table 1. In Tables 1 and 3, an entry of the form " $20 \times 5$ " in the "SOC" column means that there are 205 dimensional second-order cones, and the "ratio" denotes the ratio between the number of the second-order cones and the value of $n$. 
TABLE 4: The results for the test problems with small scale.

\begin{tabular}{|c|c|c|c|c|c|c|}
\hline \multirow{2}{*}{ Problems } & \multicolumn{3}{|c|}{ MADM } & \multicolumn{3}{|c|}{ SeDuMi } \\
\hline & Iter. & Time & Value & Iter. & Time & Value \\
\hline P21 & 326 & 10.88 & 1382.4709177 & 17 & 136.73 & 1382.4709168 \\
\hline P22 & 330 & 11.61 & 206.0023854 & 15 & 130.14 & 206.0023835 \\
\hline $\mathrm{P} 23$ & 378 & 16.78 & 7714.2171166 & 17 & 178.88 & 7714.2171243 \\
\hline $\mathrm{P} 24$ & 429 & 58.73 & 1678.7355144 & 19 & 1013.98 & 1678.7355313 \\
\hline P25 & 449 & 65.80 & 312.2865058 & 18 & 1596.53 & 312.2865057 \\
\hline P26 & 520 & 64.86 & 26103.1426757 & 19 & 1640.63 & 26103.1426826 \\
\hline $\mathrm{P} 27$ & 448 & 109.83 & 2882.0125678 & 1 & 1 & 1 \\
\hline P28 & 536 & 141.35 & 414.5181029 & l & I & I \\
\hline P29 & 566 & 150.15 & 35810.2685515 & l & l & l \\
\hline P30 & 425 & 190.92 & 6190.8005238 & 1 & 1 & 1 \\
\hline P31 & 506 & 239.69 & 801.0388729 & l & I & I \\
\hline P32 & 599 & 289.32 & 53966.4593065 & 1 & 1 & I \\
\hline P33 & 348 & 273.73 & 10471.3329318 & I & 1 & 1 \\
\hline P34 & 383 & 310.31 & 1145.2045565 & l & l & l \\
\hline P45 & 507 & 413.41 & 170350.7785126 & l & l & I \\
\hline
\end{tabular}

As is known to all, the interior-point methods have proved to be one of the most efficient class of methods for SOCP. Here the Matlab program codes for the interior-point method are designed from the software package by SeDuMi [4]. In the SeDuMi software, we set the desired accuracy parameter pars.eps $=10^{-6}$.

Let $\Delta f_{k}=\left|f\left(x^{k}\right)-f\left(x^{k-1}\right)\right|$, where $f(x)=x^{T} Q x+c^{T} x$. In the alternating direction method, we stop our algorithm when

$$
\begin{aligned}
& \max \left\{\left\|x^{k}-x^{k-1}\right\|_{2},\left\|y^{k}-y^{k-1}\right\|_{2},\left\|\lambda^{k}-\lambda^{k-1}\right\|_{2},\right. \\
& \left.\left\|\mu^{k}-\mu^{k-1}\right\|_{2}, \Delta f_{k}\right\} \leq \epsilon
\end{aligned}
$$

for $\epsilon>0$. Here we set $\beta_{1}=0.8, \beta_{2}=0.8, \gamma_{1}=\lambda_{\text {max }}(Q)+$ $0.0001, \gamma_{2}=\lambda_{\max }\left(A^{T} A\right)+0.0001$ and $\epsilon=10^{-6}$. We choose the initial point $x^{0}=e_{n}, y^{0}=e_{n}, \lambda^{0}=e_{m}$, and $\mu^{0}=e_{n}$, where $e_{n}$ is the $n$-dimensional vector of ones.

For the first set of test problems, the iteration number and average CPU time are used to evaluate the performances of the modified alternating direction method and the interiorpoint method by SeDuMi. The test results are shown in Table 2. In the Tables 2 and 4, "Time" represents the average CPU time (in seconds), and "Iter." denotes the average number of iteration. In addition, "MADM" represents the modified alternating direction method. In Table 4, "/" denotes that the method does not work in our personal computer because the method is "out of memory."

Table 2 shows that the modified alternating direction method costs less CPU time than the interior-point method by SeDuMi. But, the iteration number of the interior-point method is less than that of the modified alternating direction method.

In addition, Table 1 gives different kinds of test problem, including the problems with only one large second-order cone, such as P01, P05, P09, and P13, the problems with many small second-order cones, such as P04, P08, P12, and
P16, and the problems with one large second-order cone and some small second-order cones, such as P02, P06, P10, and P14. The test results in Table 2 show that the modified alternating direction method can solve different kinds of convex quadratic second-order cone programming problems within appropriate CPU time and accuracy.

The second set of test problems includes 15 medium scale problems, which is shown in Table 3. For the second set of test problems, the test results are shown in Table 4.

The results in Table 4 show the interior point method by SeDuMi does not work for the transformed problem (3) because of being "out of memory" in our personal computer when $n>2000$, but the modified alternating direction method is still efficient because the modified alternating direction method needs less memory space than the interiorpoint method.

In addition, we add test results of P04 and P12 in smaller criteria and with random initial points. The smaller criteria of our method is $10^{-10}$. In addition, we do one hundred experiments with the random initial point. The test results are shown in Table 5. In the SeDuMi software, we set the desired accuracy parameter pars.eps $=10^{-8}$.

Table 5 shows that the performances of MADM with random initial points are a bit better than that of MADM with fixed initial points in two different stop criteria. In addition, the number of iteration of MADM with $\epsilon=10^{-10}$ is more than that of MADM with $\epsilon=10^{-6}$, and the CPU time of MADM with $\epsilon=10^{-10}$ is longer than that of MADM with $\epsilon=10^{-6}$.

\section{Conclusion}

In the paper, a modified alternating direction method is proposed for solving convex quadratic second-order cone programming problems with bounded constraints. The proposed method does not require solving subvariational inequality 
TABLE 5: The results in smaller criteria and with random initial points.

\begin{tabular}{|c|c|c|c|c|c|c|}
\hline \multirow{2}{*}{ Problems } & \multicolumn{3}{|c|}{ MADM } & \multicolumn{3}{|c|}{ SeDuMi } \\
\hline & Iter. & Time & Value & Iter. & Time & Value \\
\hline P04 $\left(10^{-6}\right.$, random point $)$ & 275 & 0.37 & 169.24049123 & 21 & 0.88 & 169.24049003 \\
\hline P04 (10 $0^{-6}$, fixed point $)$ & 287 & 0.41 & 169.24049234 & 21 & 0.88 & 169.24049003 \\
\hline P04 $\left(10^{-10}\right.$, random point $)$ & 485 & 0.62 & 169.24049007 & 21 & 0.88 & 169.24049003 \\
\hline P04 $\left(10^{-10}\right.$, fixed point $)$ & 494 & 0.67 & 169.24049007 & 21 & 0.88 & 169.24049003 \\
\hline P12 $\left(10^{-6}\right.$, random point $)$ & 355 & 2.55 & 1904.587401 & 27 & 20.52 & 1904.587416 \\
\hline $\mathrm{P} 12\left(10^{-6}\right.$, fixed point $)$ & 367 & 2.95 & 1904.587402 & 27 & 20.52 & 1904.587416 \\
\hline P12 $\left(10^{-10}\right.$, random point $)$ & 628 & 4.37 & 1904.587409 & 27 & 20.52 & 1904.587416 \\
\hline P12 $\left(10^{-10}\right.$, fixed point $)$ & 642 & 5.20 & 1904.587408 & 27 & 20.52 & 1904.587416 \\
\hline
\end{tabular}

problems over the second cones and the bounded set. At each iteration, we only need to compute the metric projection onto the second-order cones and a projection onto the bounded set. The proposed modified method does not require secondorder information and it is easy to implement. The random simulation results show that our method can efficiently solve some convex quadratic second-order cone programming problems of vector size up to 5000 within reasonable time and accuracy by using a desktop computer.

\section{Disclosure}

This work was conducted while Xuewen Mu has been visiting Ohio University, Department of Mathematics.

\section{Conflict of Interests}

The authors declare that there is no conflict of interests regarding the publication of this paper.

\section{Acknowledgments}

The work is supported by China Scholarship Council (CSC). This work was also supported by the National Science Foundations for Young Scientists of China $(11101320,61201297)$ and the Fundamental Research Funds for the Central Universities (JB150713).

\section{References}

[1] X. Zhang, Z. Liu, and S. Liu, "A trust region SQP-filter method for nonlinear second-order cone programming," Computers \& Mathematics with Applications, vol. 63, no. 12, pp. 1569-1576, 2012.

[2] H. Kato and M. Fukushima, "An SQP-type algorithm for nonlinear second-order cone programs," Optimization Letters, vol. 1, no. 2, pp. 129-144, 2007.

[3] X. Y. Zhao, A semismooth Newton-CG augmented Lagrangian method for large scale linear and convex quadratic SDPs [Ph.D. thesis], National University of Singapore, 2009.

[4] J. F. Sturm, "Using SeDuMi 1.02, a MATLAB toolbox for optimization over symmetric cones," Optimization Methods and Software, vol. 11, no. 1-4, pp. 625-653, 1999.
[5] R. H. Tütüncü, K. C. Toh, and M. J. Todd, "Solving semidefinitequadratic-linear programs using SDPT3," Mathematical Programming, vol. 95, pp. 189-217, 2003.

[6] S. H. Schmieta and F. Alizadeh, "Associative and Jordan algebras, and polynomial time interior-point algorithms for symmetric cones," Mathematics of Operations Research, vol. 26, no. 3, pp. 543-564, 2001.

[7] S. H. Schmieta and F. Alizadeh, "Extension of primal-dual interior point algorithms to symmetric cones," Mathematical Programming, vol. 96, no. 3, pp. 409-438, 2003.

[8] R. D. Monteiro and T. Tsuchiya, "Polynomial convergence of primal-dual algorithms for the second-order cone program based on the MZ-family of directions," Mathematical Programming, vol. 88, no. 1, pp. 61-83, 2000.

[9] J. Eckstein and D. P. Bertsekas, An Alternating Direction Method for Linear Programming, LIDS-P, Laboratory for Information and Decision Systems, Massachusetts Institute of Technology, Cambridge, Mass, USA, 1967.

[10] Z. Yu, "Solving semidefinite programming problems via alternating direction methods," Journal of Computational and Applied Mathematics, vol. 193, no. 2, pp. 437-445, 2006.

[11] J. Malick, J. Povh, F. Rendl, and A. Wiegele, "Regularization methods for semidefinite programming," SIAM Journal on Optimization, vol. 20, no. 1, pp. 336-356, 2009.

[12] P. Tseng, "Alternating projection-proximal methods for convex programming and variational inequalities," SIAM Journal on Optimization, vol. 7, no. 4, pp. 951-965, 1997.

[13] Y. Wang, J. Yang, W. Yin, and Y. Zhang, "A new alternating minimization algorithm for total variation image reconstruction," SIAM Journal on Imaging Sciences, vol. 1, no. 3, pp. 248-272, 2008.

[14] J. Yang, Y. Zhang, and W. Yin, "An efficient TVL1 algorithm for deblurring multichannel images corrupted by impulsive noise," SIAM Journal on Scientific Computing, vol. 31, no. 4, pp. 28422865, 2009.

[15] J. Sun and S. Zhang, "A modified alternating direction method for convex quadratically constrained quadratic semidefinite programs," European Journal of Operational Research, vol. 207, no. 3, pp. 1210-1220, 2010.

[16] Z. Wen, D. Goldfarb, and W. Yin, "Alternating direction augmented Lagrangian methods for semidefinite programming," Mathematical Programming Computation, vol. 2, no. 3-4, pp. 203-230, 2010.

[17] U. Faraut and A. Koranyi, Analysis on Symmetric Cone, Oxford University Press, New York, NY, USA, 1994. 
[18] J. V. Outrata and D. Sun, "On the coderivative of the projection operator onto the second-order cone," Set-Valued Analysis, vol. 16, no. 7-8, pp. 999-1014, 2008.

[19] L. C. Kong, L. Tuncel, and N. H. Xiu, "Clarke generalized jacobian of the projection onto symmetric cones," Set-Valued and Variational Analysis, vol. 17, pp. 135-151, 2009.

[20] D. Kinderlehrer and G. Stampacchia, An Introduction to Variational Inequalities and their Applications, Academic Press, New York, NY, USA, 1980. 


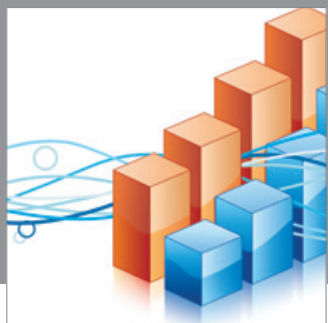

Advances in

Operations Research

mansans

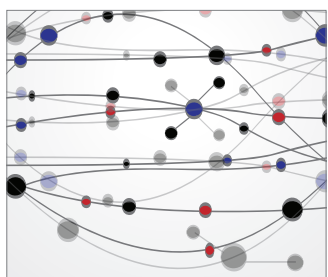

The Scientific World Journal
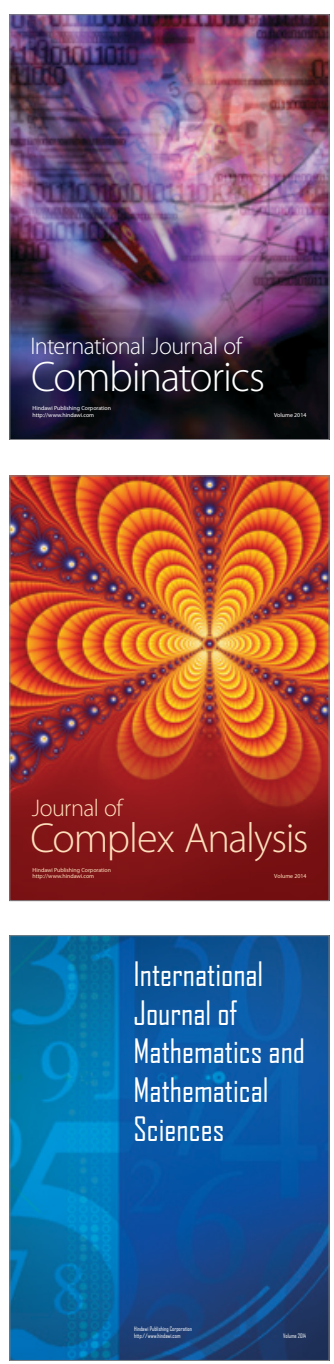
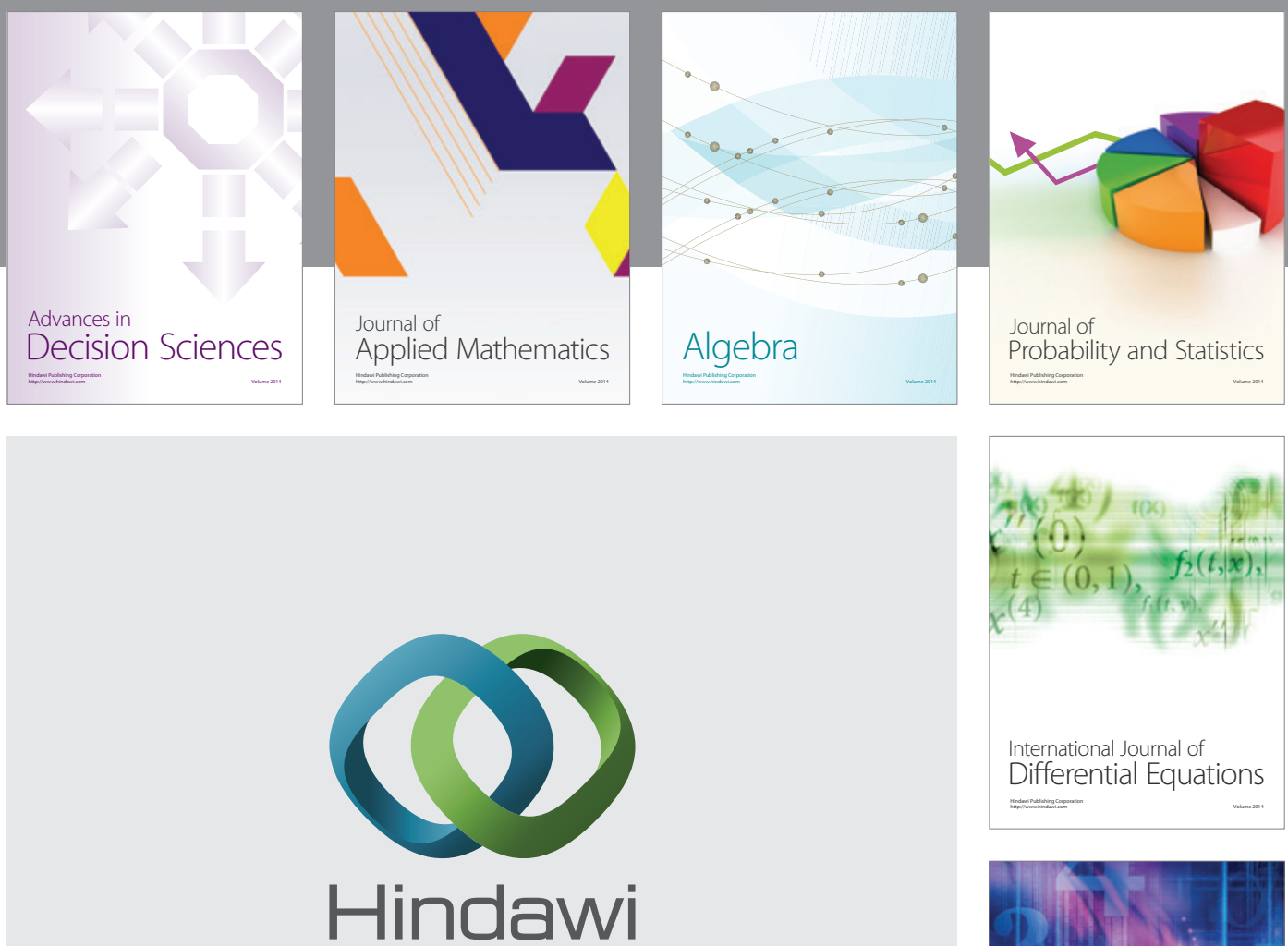

Submit your manuscripts at http://www.hindawi.com
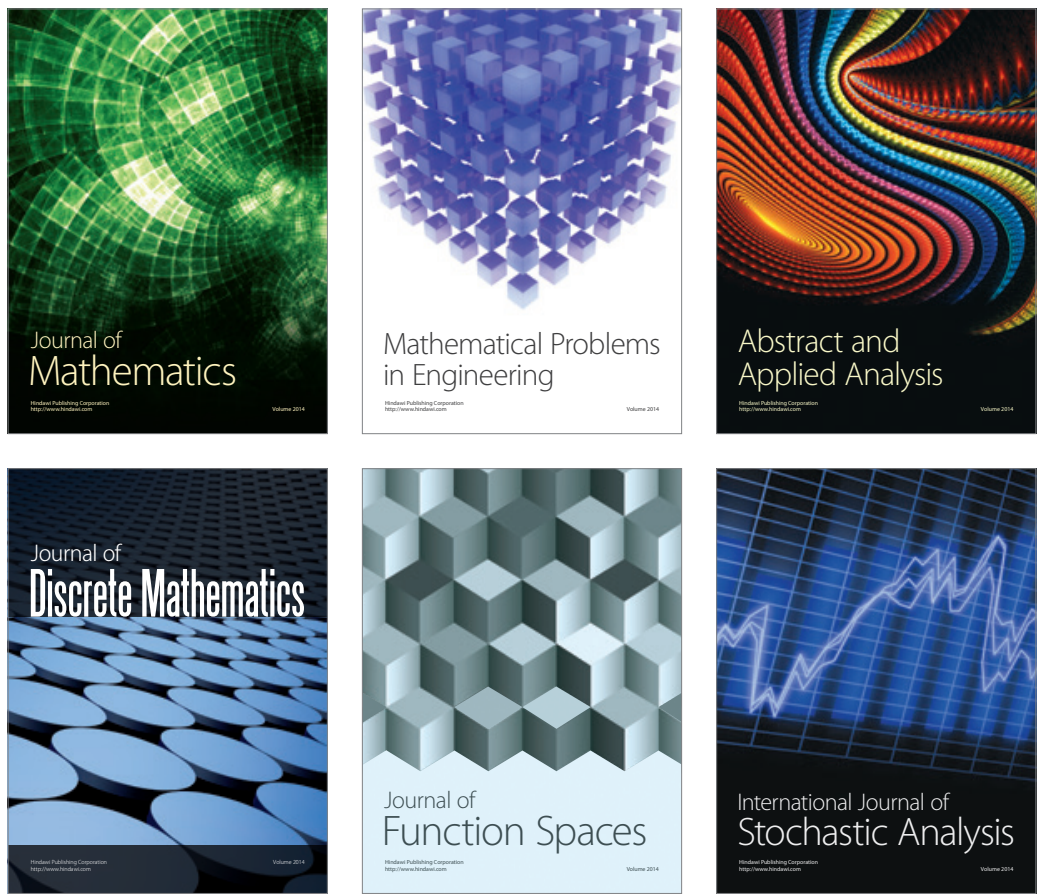

Journal of

Function Spaces

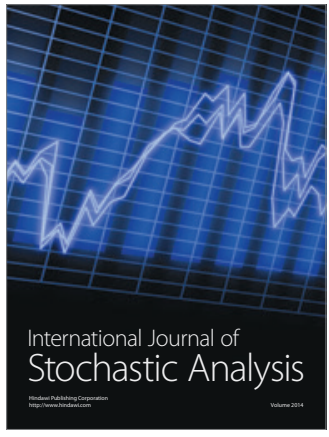

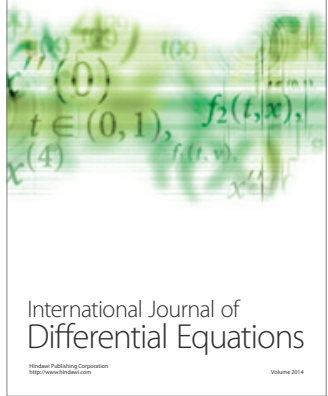
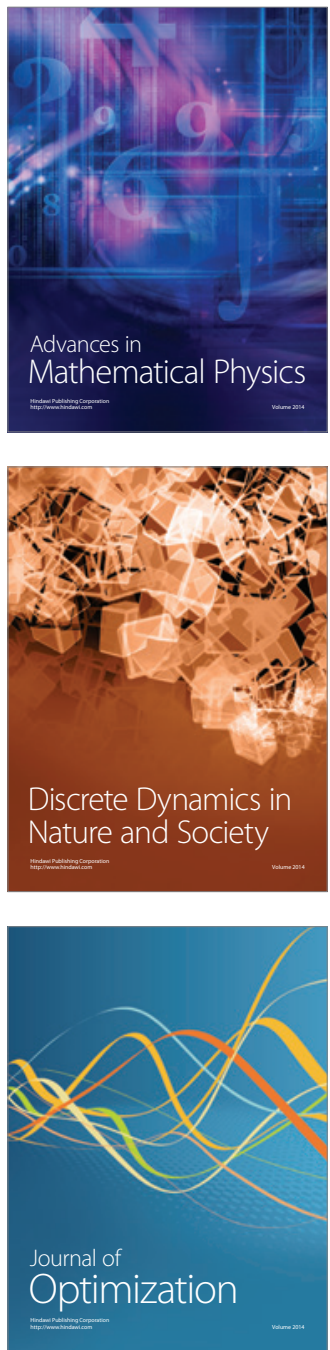